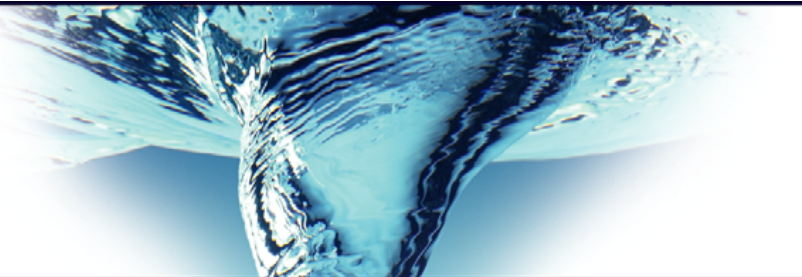

\title{
Lower branch equilibria in Couette flow: the emergence of canonical states for arbitrary shear flows
}

\author{
H. M. Blackburn ${ }^{1} \dagger$, P. Hall ${ }^{2}$ and S. J. Sherwin ${ }^{3}$ \\ ${ }^{1}$ Department of Mechanical and Aerospace Engineering, Monash University, Victoria 3800, Australia \\ ${ }^{2}$ Department of Mathematics, Imperial College London, South Kensington, London SW7 2AZ, UK \\ ${ }^{3}$ Department of Aeronautics, Imperial College London, South Kensington, London SW7 2AZ, UK
}

(Received 4 February 2013; revised 27 April 2013; accepted 16 May 2013)

We consider the development of nonlinear three-dimensional vortex-wave interaction equilibria of laminar plane Couette flow for a range of spanwise wavenumbers. The results are computed using a hybrid approach that captures the required asymptotic structure while at the same time providing a direct link with full numerical calculations of equilibrium states. Each equilibrium state consists of a streak flow, a roll flow and a wave propagating on the streak. Direct numerical simulations at finite Reynolds numbers using initial conditions constructed from these parts confirm that the scheme generates equilibrium solutions of the Navier-Stokes equations. Consideration of the form of the vortex-wave interaction equations in the highspanwise-wavenumber limit predicts that for small wavelengths the equilibria take on a self-similar structure confined near the centre of the flow. These states feel no influence from the walls, and lead to a class of canonical states relevant to arbitrary shear flows. These predictions are supported by an analysis of computational results at increasing values of the spanwise wavenumber. For the wave part of these new canonical states, it is shown that the mass-specific kinetic energy density per unit wavenumber scales with the $-5 / 3$ power of the wavenumber.

Key words: boundary layer stability, critical layers, transition to turbulence

\section{Introduction}

Understanding the fundamental mechanisms at work in turbulent shear flows is one of the outstanding problems of classical mechanics. Two facets of this problem that exist at opposite ends of the Reynolds-number spectrum are the mechanism of

$\dagger$ Email address for correspondence: Hugh.Blackburn@monash.edu 


\section{H. M. Blackburn, P. Hall and S. J. Sherwin}

transition in flows such as Hagen-Poiseuille flow and plane Couette flow, which are linearly stable and whose instability mechanisms have not yielded to analysis with linear tools, and the universal inertial-range scaling of turbulence kinetic energy with wavenumber first proposed by Kolmogorov (1941), which applies at very high Reynolds numbers. In recent years much interest in the dynamics of shear flows has come through the discovery of three-dimensional nonlinear equilibrium states (Nagata 1990; Waleffe 1997, 2001; Faisst \& Eckhardt 2003; Wedin \& Kerswell 2004; Wang, Gibson \& Waleffe 2007; Gibson, Halcrow \& Cvitanovic 2009). These equilibria have been shown to act either as 'edge states' which locally divide the basin of attraction between relaminarized or turbulent outcomes ('lower branch' equilibria) or as 'organizing centres' about which the flow slowly cycles during the approach to turbulence ('upper branch' equilibria) (Wang et al. 2007). The physical mechanism supporting the self-sustaining lower branch states was proposed (Waleffe 1997) as a tripartite coupled nonlinear system in which the dominant component is two-dimensional high- and low-speed streaks of $O(1)$ appearing on the underlying streamwise flow; these streaks are driven by weaker two-dimensional rolls, which in turn are driven nonlinearly by the divergence of streamwise-average Reynolds stresses of still weaker three-dimensional waves that are neutrally stable eigenmodes of the streak flow. Waleffe (1997), in dealing with plane Couette flow, supplied no direct means of determining the required amplitudes of the roll and wave components relative to the streak flow, but in Wang et al. (2007), where nonlinear equilibria were found directly using Newton's method applied to full Navier-Stokes solutions, it was observed that the strength of the rolls fitted an $O\left(R^{-1}\right)$ dependence (where Reynolds number $R$ is based on half the relative wall speeds and their semi-distance d) while the strength of the three-dimensional waves fitted $O\left(R^{-0.9}\right)$. On this basis it was suggested by Wang et al. (2007) that an asymptotic theory for the lower branch equilibria appeared feasible.

The appropriate asymptotic structure for these kinds of interactions in shear flows had been laid down some years earlier and referred to as a 'vortex-wave interaction' (VWI) theory (Hall \& Smith 1988, 1991). This theory effectively takes $R$ out of consideration and reduces an unsteady three-dimensional Navier-Stokes problem to a steady two-dimensional Navier-Stokes problem coupled to an advection-diffusion equation and a wave equation. Recognition that the solutions of Nagata (1990), Waleffe (1997), Faisst \& Eckhardt (2003), Wedin \& Kerswell (2004), Wang et al. (2007) and Gibson et al. (2009) were finite- $R$ analogues of VWI states stimulated application of the theory to plane Couette flow (Hall \& Sherwin 2010). Outcomes of that asymptotic approach agreed remarkably with the 'lower branch' equilibria found in Wang et al. (2007) and explicitly provide asymptotic scaling relations $R^{-1}$ and $R^{-11 / 12=-0.91 \overline{6}}$ for roll and wave components. We note that the sum of the streak and roll flows constitutes the vortex engaged in vortex-wave interaction.

Our aim here is to extend the results of Hall \& Sherwin (2010) to a large range of streamwise $(\alpha)$ and spanwise $(\gamma)$ wavenumbers and show how the key components of the interaction perform. In order to simplify the computation associated with the asymptotic approach, we introduce a new method that can be seen as a direct hybrid of the streak-roll-wave coupling described originally in Waleffe (1997) with the asymptotic scaling of components used in Hall \& Sherwin (2010). This hybrid approach may be considered as a regularization of the VWI equations, and used for quite general shear flows. Direct numerical simulations (DNS) are also carried out to investigate the temporal evolution of VWI states, and their energetics confirm the scaling approach employed and the status of the VWI equilibria as edge states. In 


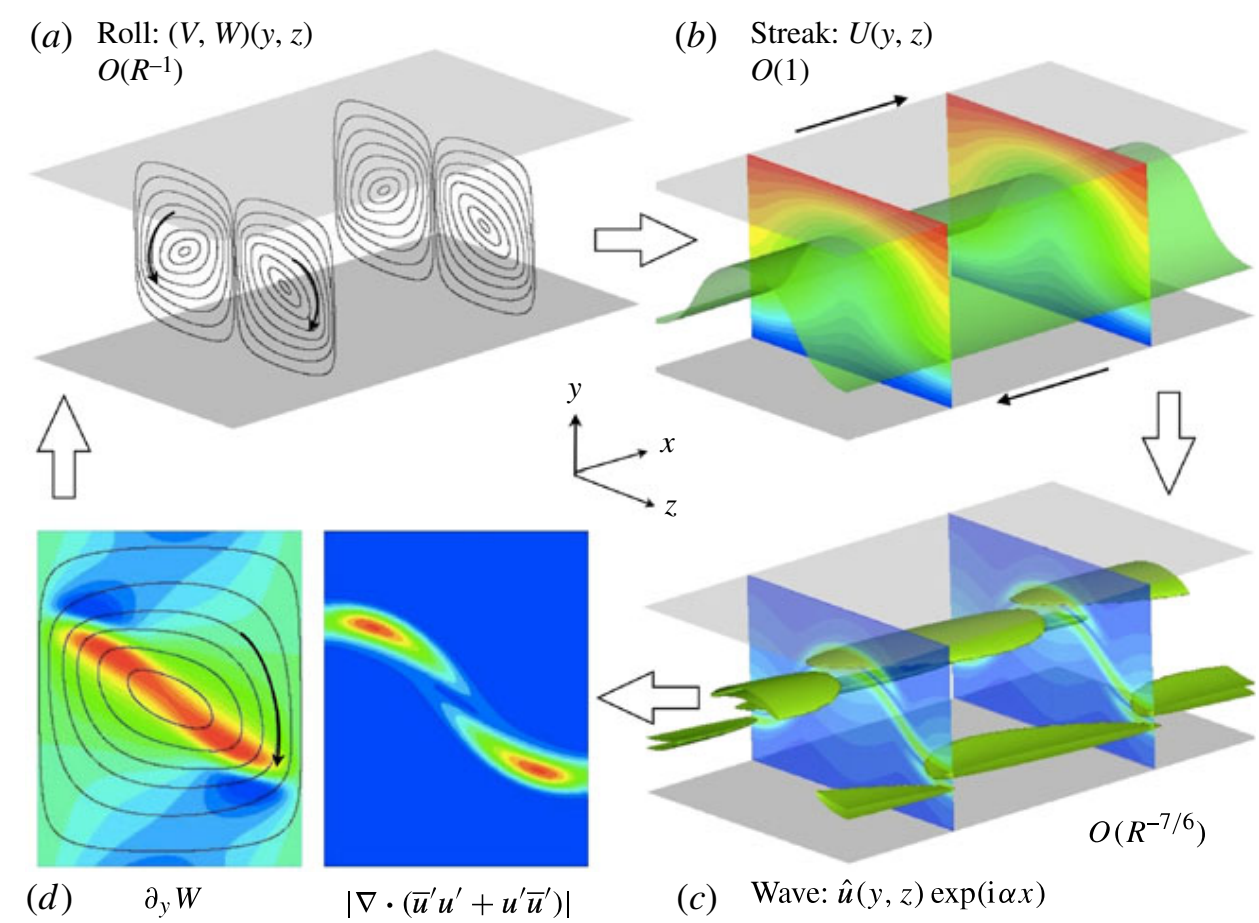

FIgURE 1. Vortex-wave interaction in plane Couette flow. $(a)$ Roll flow $(V, W)(y, z)$ drives (b) streak flow $U(y, z)$. Linearizing the Navier-Stokes equations about $U(y, z)$, we seek a neutral and stationary wave eigenfunction $(c)$ that concentrates near the streak's critical layer. (d) Streamwise-average Reynolds stresses of the wave system at finite amplitude balance dissipation in the roll-streak flow to couple the whole problem at equilibrium.

the high- $\gamma$ limit we find that VWI becomes localized at the centre of the channel with the walls playing no role, and the equilibria then become self-similar in structure. This leads to a new canonical state relevant to arbitrary shear flows. Finally, we show that, for this canonical state, kinetic energy per unit wavenumber scales with the $-5 / 3$ power of wavenumber. Suggestively, though perhaps coincidentally, this is the same scaling derived by Kolmogorov (1941) for the equilibrium wavenumber range of homogeneous isotropic turbulence.

\section{The vortex-wave interaction equations and their solution}

We begin by writing down the VWI equations of Hall \& Sherwin (2010) and then describe a new hybrid method for the asymptotic solution, which is relatively straightforward to implement, and which connects with the full numerical Navier-Stokes-based approach of Waleffe (1997). As in those works, the vehicle of exposition is incompressible plane Couette flow (see figure 1). Equilibrium states are solutions of a nonlinear eigenproblem, whose eigenvalue $\rho(\alpha, \gamma)$ is a measure of the scaled wave amplitude required to maintain equilibrium, with streamwise and spanwise wavenumbers as parameters. The mechanism by which the wave drives the roll is an analogue of acoustic streaming, with the wave essentially trapped in a waveguide of thickness $R^{-1 / 3}$ surrounding its critical layer. Unlike typical acoustic streaming problems, the fact that the edge of the 'waveguide' is a fluid region means that, rather than introducing a jump in mean flow velocity, the streaming induces a jump in stress. This stress jump, corresponding to the integral across the critical layer of divergence 


\section{H. M. Blackburn, P. Hall and S. J. Sherwin}

of wave Reynolds stress obtained via a streamwise average along the wave, drives the roll field. Thus the underlying mechanism in VWI is the same as was described later by Waleffe (1997), though asymptotic scaling is made explicit in the formulation, with the role played by the critical layer brought to the fore. The introduction of asymptotics also produces partial decoupling of the streak, roll and wave components of the solution, as outlined below.

The momentum and continuity equations are

$$
\mathrm{D} \boldsymbol{u} / \mathrm{D} t=-\nabla p+R^{-1} \nabla^{2} \boldsymbol{u} \quad \text { with } \nabla \cdot \boldsymbol{u}=0 .
$$

Adopting Cartesian coordinates $(x, y, z)$, considering $\boldsymbol{u}=(u, v, w)$ as periodic in $x$ and $z$, with corresponding wavelengths $\left(L_{x}=2 \pi / \alpha, L_{z}=2 \pi / \gamma\right)$, and, following Hall \& Sherwin (2010), we note that (2.1) admits equilibrium high- $R$ solutions of the form

$$
\begin{aligned}
\boldsymbol{u}= & {\left[U(y, z), V(y, z) R^{-1}, W(y, z) R^{-1}\right]+\cdots } \\
& +\rho R^{-7 / 6} \boldsymbol{u}^{\prime}(x, y, z, t)+\text { c.c. }+\cdots, \\
p= & P(y, z) R^{-2}+\rho R^{-7 / 6} p^{\prime}(x, y, z, t)+\text { c.c. }+\cdots,
\end{aligned}
$$

where $U(y, z)$ is a two-dimensional streak flow, $\boldsymbol{V}=(V, W)(y, z)$ is a twodimensional roll flow, and $\boldsymbol{u}^{\prime}=\widehat{\boldsymbol{u}}(y, z) \exp [\mathrm{i} \alpha(x-c t)], p^{\prime}=\widehat{p}(y, z) \exp [\mathrm{i} \alpha(x-c t)]$ is a three-dimensional wave system with amplitude $\rho$. The $R^{-7 / 6}$ scaling of the wave is fixed by the condition that the wave generates Reynolds stresses in the critical layer of the streak which induce a stress jump of $O(1)$ in the roll flow.

Inserting (2.2) into (2.1) and neglecting higher harmonics of the wave, the set of momentum equations can be decomposed into those for the $O(1)$ twodimensional streak,

$$
V \partial_{y} U+W \partial_{z} U=\nabla^{2} U
$$

the $O\left(R^{-1}\right)$ two-dimensional roll,

$$
\begin{aligned}
V \partial_{y} V+W \partial_{z} V & =-\partial_{y} P+\nabla^{2} V+F_{y}, \\
V \partial_{y} W+W \partial_{z} W & =-\partial_{z} P+\nabla^{2} W+F_{z}, \\
\partial_{y} V+\partial_{z} W & =0,
\end{aligned}
$$

and a three-dimensional wave. In (2.4), $\boldsymbol{F}=\left(F_{y}, F_{z}\right)$ has a delta-function-like behaviour around the critical layer, induced by the interaction of the wave with itself inside the critical layer. Within the critical layer, the wave components are $O\left(R^{-5 / 6}\right)$. However, away from the critical layer, the three-dimensional wave is $O\left(R^{-7 / 6}\right)$ and governed by

$$
\begin{aligned}
\partial_{t} u^{\prime}+U \partial_{x} u^{\prime}+v^{\prime} \partial_{y} U+w^{\prime} \partial_{z} U & =-\partial_{x} p^{\prime}+R^{-1} \nabla^{2} u^{\prime}, \\
\partial_{t} v^{\prime}+U \partial_{x} v^{\prime} & =-\partial_{y} p^{\prime}+R^{-1} \nabla^{2} v^{\prime}, \\
\partial_{t} w^{\prime}+U \partial_{x} w^{\prime} & =-\partial_{z} p^{\prime}+R^{-1} \nabla^{2} w^{\prime}, \\
\partial_{x} u^{\prime}+\partial_{y} v^{\prime}+\partial_{z} w^{\prime} & =0 .
\end{aligned}
$$

In Hall \& Sherwin (2010), the viscous form of the wave equations was used in order to take care of the singularity at the critical layer. However, here, since we do not explicitly determine the asymptotic structure in the critical layer, the Reynolds number is retained in order to obtain the correct asymptotic structure, which then feeds into the generation of $\boldsymbol{F}$. Thus in the present formulation the retention of the viscous terms 


\section{Lower branch equilibria in Couette flow}

can be viewed as an alternative regularization of the inviscid wave equations and a means to automatically generate the correct structure in the critical layer and for jumps across it. These points are revisited in $\S \S 2.1$ and 2.2. The solution to (2.5) requires normalization to close the set of equations, for which we choose

$$
\int \bar{p}^{\prime} p^{\prime} \mathrm{d} y \mathrm{~d} z=L_{y} L_{z}
$$

where $\bar{p}^{\prime}$ is the complex conjugate of wave pressure $p^{\prime}$.

For equilibrium solutions, one seeks neutral wave modes where $\operatorname{Im}(c)=0$. We note that the steady roll flow (2.4) is not directly coupled to the streak flow (2.3), that the streak flow is the solution to a steady advection-diffusion equation driven by the roll, and that after the above decomposition both are solved at unit Reynolds number. Since the streak flow is $O(R)$ larger than the roll flow, only linear advection terms derived from the streak flow are included in the wave system (2.5).

It remains for us to close the problem by writing down the coupling of the wave to the roll flow, formally via stress jumps derived asymptotically from the wave pressure field - the approach taken by Hall \& Sherwin (2010), outlined in $\S 2.1$ - or computed directly from the velocity field of the wave - the hybrid approach of $\S 2.2$.

\subsection{Asymptotic closure of the vortex-wave equations}

When the viscous terms in (2.5) are neglected, as required by the asymptotic analysis, the inviscid equations for the wave pressure $p^{\prime}=\widehat{p}(y, z) \exp [\mathrm{i} \alpha(x-c t)]$ of the neutrally stable wave eigenmode satisfy (Hall \& Horseman 1991)

$$
\nabla \cdot\left(\nabla p^{\prime} /(U-c)\right)=0 .
$$

This equation breaks down at the critical layer at $y=f(z)$ where $U=c$. The roll and wave equations then need to be solved in this layer in the manner described by Hall \& Smith (1991) and Hall \& Sherwin (2010). The roll equation is driven in the critical layer by the Reynolds stresses associated with the wave. Analysis presented by Hall \& Smith (1991) showed that (2.4) must be solved with the following jumps in roll stresses across the critical layer:

$$
\begin{aligned}
{\left[\partial_{n} V^{s}(n, s)\right]_{-}^{+} } & =n_{0} \rho^{2} \alpha^{-5 / 3} \partial_{s}\left(\mu^{-5 / 3}\left|\partial_{s} \widehat{p}\right|^{2}\right), \\
{[P]_{-}^{+} } & =-n_{0} \rho^{2}(\alpha \mu)^{-5 / 3} \Lambda_{0}\left|\partial_{s} \widehat{p}\right|^{2} .
\end{aligned}
$$

Here $V^{s}(n, s)$ is the roll velocity tangential to the critical layer in the $y-z$ plane, $(n, s)$ are normal and tangential coordinates along the critical layer, $\Lambda_{0}$ is the layer curvature, $\mu=\partial_{n} U$ and $n_{0}=2 \pi(2 / 3)^{2 / 3} \Gamma(1 / 3)$. Note that these jump conditions are determined in terms of the wave pressure. The wave pressure is finite at the critical layer and can be found by solving the inviscid pressure equation, and directly accounting for discontinuities in the derivatives of the pressure at higher order, or by regularizing the equations and solving (2.5).

In Hall \& Sherwin (2010) the jump conditions were enforced through a deltafunction-type regularization of forcing terms $\left(F_{y}, F_{z}\right)$ in (2.4). That regularization involved the introduction of a parameter $\xi$ that relates to the thickness of the approximation to the delta-function. As the wave pressure field is relatively smooth, solutions to the coupled system become independent of the regularization parameter $\xi$ and the regularized Reynolds number, used in finding neutral solutions to (2.5), provided that adequate resolution is retained near the critical layer. In this approach, however, one does need to track and resolve solutions near to the critical layer, not 


\section{H. M. Blackburn, P. Hall and S. J. Sherwin}

only so that the jumps in (2.8) may be computed from the wave pressure field but also so that the jumps may be adequately approximated with delta-function-like shapes.

\subsection{Closure via a hybrid computational approach}

In the hybrid approach, the roll forcing $\left(F_{y}, F_{z}\right)$ is directly computed from the Reynolds stresses of the wave velocity field, with scaling appropriate for the wave amplitude required in the critical layer as deduced from the asymptotic VWI theory (Hall \& Sherwin 2010):

$$
\boldsymbol{F}=\rho^{2} R^{-1 / 3}\left[\nabla \cdot\left(\overline{\boldsymbol{u}}^{\prime} \boldsymbol{u}^{\prime}+\boldsymbol{u}^{\prime} \overline{\boldsymbol{u}}^{\prime}\right)\right],
$$

where $\overline{\boldsymbol{u}}^{\prime}$ is the complex conjugate of $\boldsymbol{u}^{\prime}$, which again is obtained from the neutrally stable wave eigenmode. As shown in the right-hand panel of figure $1(d), \boldsymbol{F}$ becomes localized around the critical layer where $U=c$. We demonstrate below that the eigenvalue $\rho$ in (2.9) becomes independent of the regularization Reynolds number $R$, as required.

In the limit $R \rightarrow \infty$ in (2.5) the forcing will recover the asymptotic value and therefore, in the critical layer region where the advection terms in (2.4) become negligible by comparison, the divergence of the wave Reynolds stress is balanced by the roll stress, i.e.

$$
\boldsymbol{F}=\nabla P-\nabla^{2} \boldsymbol{V}
$$

A potential advantage of this hybrid closure arises from the fact that the roll forcing can be computed directly from the wave eigenmode velocity field without recourse to (2.8) and subsequent reconstruction of forcing with delta-function-like regularization. Although formally this means that no special treatment is required near the critical layer, it also implies that the critical layer velocity structure has to be adequately resolved, which we have observed can become computationally demanding for large $\gamma$ values. The hybrid method is therefore computationally convenient to implement since it uses the immediately available wave velocity from (2.5), but has the limitation that, to achieve the asymptotic high- $R$ limit, one must resolve the critical layer velocity structure rather than being able to use the properties of the smoother wave pressure. We have used the hybrid method to compute the VWI solutions presented herein.

\subsection{Iterative solution of the coupled problem}

One method for obtaining a solution to the system (2.3)-(2.6) and (2.9) is iteration of the loop illustrated in figure 1 while adjusting one parameter $(\alpha, \gamma$ or $\rho)$ to achieve stationary equilibrium $(c=0)$ for the leading linear wave eigenmode. The restriction to both neutral and stationary waves facilitates comparison to previous works dealing with lower branch equilibria (e.g. Wang et al. 2007; Hall \& Sherwin 2010), though perhaps only neutrality is required; we have yet to examine this point in depth. We have found that it is typically helpful in seeking neutral, stationary solutions to also impose the symmetry condition $\boldsymbol{F}(y, z)=-\boldsymbol{F}(-y,-z+\pi / \gamma)$.

When applied to plane Couette flow, this solution methodology produces an $R$ independent problem that describes lower branch equilibrium states, which was also a central result of Hall \& Sherwin (2010). As highlighted in $\S 4$, at higher $\gamma$ values one requires an increasingly large regularization Reynolds number to obtain the correct critical layer structure, which leads to numerical challenges in resolving the layer structure and consequently also in obtaining iterative solutions that agree with the asymptotic structure. 


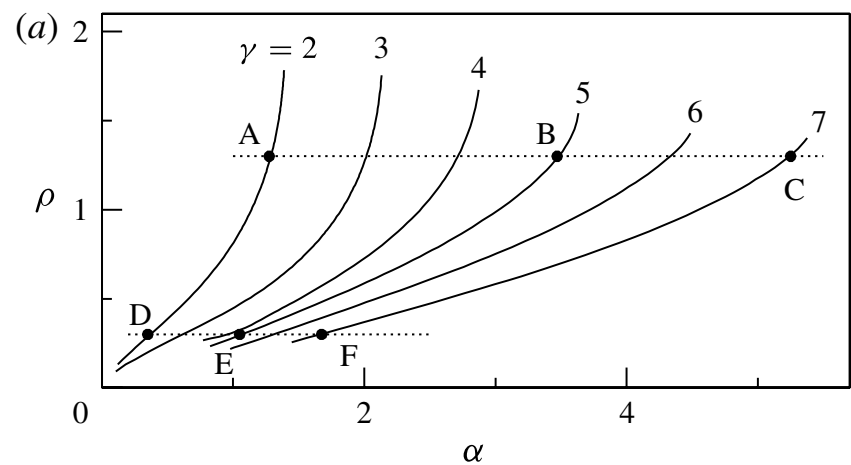

(b)

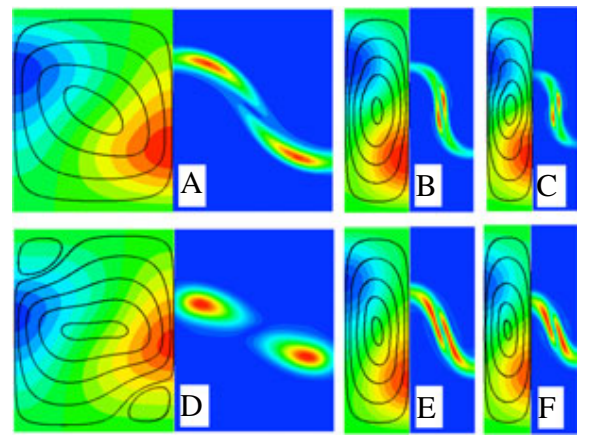

FIGURE 2. (a) Nonlinear eigenvalues $\rho=\rho(\alpha, \gamma)$ for plane Couette flow. (b) Flow visualizations in each panel $\mathrm{A}-\mathrm{F}$ show (left) contours of perturbation streak speed $U$ from background plane Couette flow with roll streamlines superimposed, and (right) contours of $|\boldsymbol{F}|$.

$\begin{array}{llllll}R & 1000 & 2000 & 5000 & 10000 & 20000 \\ \rho & 1.219 & 1.187 & 1.173 & 1.170 & 1.172\end{array}$

TABLE 1. Convergence of wave amplitude $\rho$ with regularization Reynolds number used in solving the system $(2.5 a-d)$ with the hybrid closure appproach of $\S 2.2$, at $\gamma=2$, $\alpha=1.235$.

\section{Numerical method results}

Figure $1(d)$ illustrates the idea that, when $R$ is sufficiently high, $\boldsymbol{F}$ will be negligible except near the critical layer, where it produces the required stress jumps. In our hybrid approach, $R$ may best be thought of as a regularization parameter used in solving (2.5), with the appropriate Reynolds number scaling otherwise implicit in the decomposition of the solution into streak, roll and wave components. Table 1 shows that, at fixed $(\alpha, \gamma)$, the value of wave amplitude $\rho$ becomes independent of regularization Reynolds number at moderate computational $R$, confirming that the hybrid method generates asymptotic ( $R$-independent) solutions for the nonlinear eigenvalue $\rho$.

Neutral wave solutions may be characterized by the relationship between parameters $\rho=\rho(\alpha, \gamma)$, which we illustrate in figure 2. With increasing $\gamma$, the critical layer and wave energy become more localized near the centre of the domain (a point we will return to below) and it consequently becomes necessary to use larger values of computational $R$ in (2.5) in order to obtain $R$-independent values of $\rho$. The outcomes for $\rho$ versus $\alpha$ are, to within graphical accuracy, those computed (using the closure outlined in $\S 2.1$ above) by Hall \& Sherwin (2010) at $\gamma=2$, the only value dealt with there. As found by Hall \& Sherwin, the curves could not be continued for sufficiently high $\alpha$, where the iterations failed to converge. Investigation of the results towards the end of the curves did not suggest the development of a singularity in the solution, and the failure to converge is probably associated with the wave's critical layer becoming multi-valued in $y$ at a fixed $z$. The upper limits for $\alpha$ are consistent with computationally determined minimum box sizes required for sustained turbulence in plane Couette flow (Hamilton, Kim \& Waleffe 1995). At lower values of $\alpha$ the curves all approach the origin and, though convergence for small $\alpha$ was not attained, 


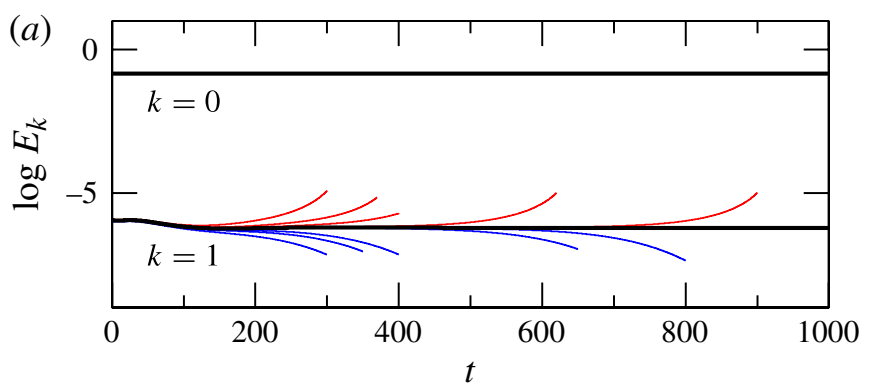

(b)

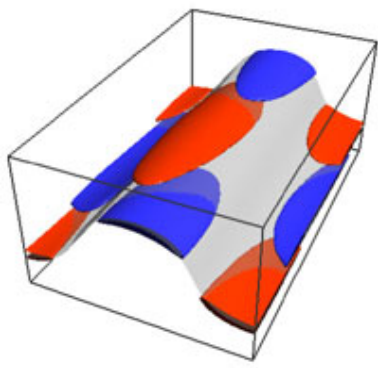

FIgURE 3. (a) Temporal evolution of kinetic energies $E_{k}$ in the leading ( $k=0$, roll-streak) and the fundamental $(k=1$, wave) streamwise Fourier modes for DNS at $\gamma=2, \alpha=1.235$ and $R=5000$. (b) A snapshot of the flow illustrated with a translucent streak critical layer and solid isosurfaces showing equal \pm values of spanwise velocity component. See also supplementary movie 1 available at http://dx.doi.org/10.1017/jfm.2013.254.

the curves are expected to enter following the $\rho \sim \alpha^{5 / 6}$ law established in Hall \& Sherwin (2010).

Figure 3 illustrates outcomes for DNS at $R=5000$. Initial conditions for these calculations were composed from streak, roll and wave components obtained using the hybrid approach outlined above, then recombined using expansion (2.2a). The simulations included only a single streamwise wave harmonic. With initial wave amplitude $\rho$ predicted via the hybrid method, the system decays to the onedimensional Couette state, but tuning $\rho$ to slightly larger values, we observe that the system reaches a near-equilibrium three-dimensional state at large times. For still larger initial wave amplitudes, the system diverges to a chaotic state. We note the similarity of behaviour to that observed elsewhere in the computation of 'edge states' (Schneider \& Eckhardt 2009). When in self-sustaining state, the relative amount of wave energy is almost exactly the same as required by the neutral solution of (2.5), and the form of the DNS solution is difficult to visibly distinguish from the initial conditions.

\section{The large- $\gamma$ limit}

\subsection{The emergence of a new class of canonical shear flow equilibria}

Figure 4 demonstrates that, as $\gamma$ becomes large, not only does the roll forcing $\boldsymbol{F}$ concentrate around the critical layer, but also the vertical extent of that region becomes proportional to the domain width $L_{z}$. As a result, the solution effectively becomes independent of the constraint provided by the walls as $\gamma$ increases. This trend can also be asymptotically observed in the VWI formulation by rescaling the problem based on $L_{z}\left(\propto \gamma^{-1}\right)$ rather than the semi-height of the channel. Based on the assumption that, as the spanwise wavenumber becomes large, the interaction concentrates into a rectangular box with sides of length $O\left(\gamma^{-1}\right)$, we now deduce the behaviour of the wave amplitude $\rho$ in that limit and check that it is consistent with our calculations.

In a rectangular box of length $O\left(\gamma^{-1}\right)$ we first observe that underlying Couette flow speed is of order $L_{z} \simeq \gamma^{-1}$, and so the roll equations will then remain similar to the nonlinear equations (2.4) if we take $V$ and $W$ to be of $O(\gamma)$. The roll and streak rescale in opposite ways because the streak scales on a typical streamwise velocity of the unperturbed flow whereas the roll scales on the viscous length scale defined in terms of the viscosity divided by the box size. Since the roll pressure scales on the 
(a)

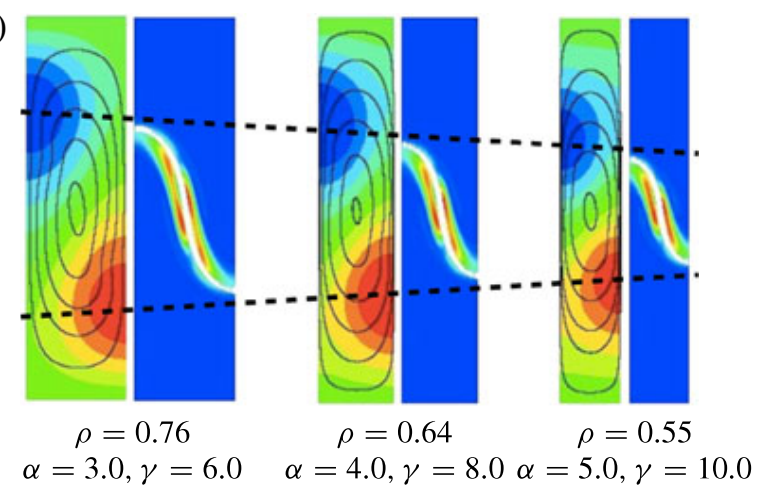

(b)

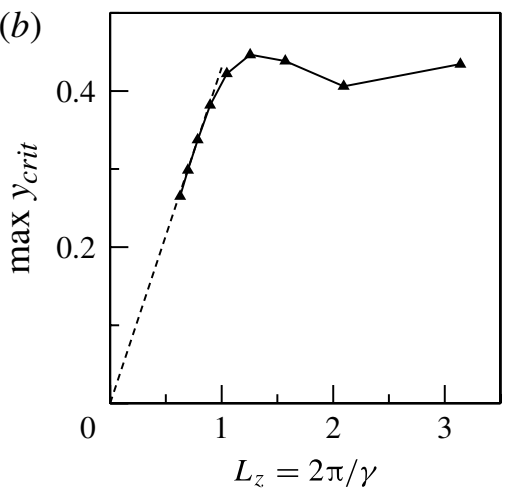

FIGURE 4. For spanwise wavenumber $\gamma \gg 1$, VWI theory predicts that the vertical extent of the critical layer should fall inversely with $\gamma$, as shown here for fixed $\alpha / \gamma=0.5$. Flow visualization features as for figure $2(b)$, with critical layer location superimposed over $|\boldsymbol{F}|$.

square of the roll velocity, it must now become $O\left(\gamma^{2}\right)$. Therefore if we take account of these rescalings in (2.4) by making the transformations

$$
\left(\partial_{x}, \partial_{y}, \partial_{z}\right) \rightarrow \gamma\left(\partial_{x}, \partial_{y}, \partial_{z}\right) \text { and }(U, V, W, P) \rightarrow\left(\gamma^{-1} U, \gamma V, \gamma W, \gamma^{2} P\right),
$$

then the roll equations (2.4) remain invariant.

In evaluating the $(\rho, \alpha, \gamma)$ data of $\S 3$, we have effectively enforced the jump conditions (2.8) through the hybrid computational method. If we now apply the above transformations on these jump conditions, we can explicitly identify their dependence on $\gamma$. First we note that the effect of these rescalings is to make the left-hand side terms of (2.8) of $O\left(\gamma^{2}\right)$. We next observe that the normal shear, $\mu$, of the streak at the critical layer remains $O(1)$ under the rescaling, but the partial derivatives along the critical layer are of $O(\gamma)$, and so $\partial_{s} \rightarrow \gamma \partial_{s}$, while under the rescaling $\alpha \rightarrow \gamma \alpha$. Therefore, both sides of (2.8) will remain in balance if $\gamma^{2} \sim \gamma^{4 / 3} \rho^{2} p^{2}$, which necessarily implies $\rho^{2} p^{2} \sim \gamma^{2 / 3}$.

Finally we note that the pressure normalization (2.6) we have adopted in $\S 2$ depends on the channel height $L_{y}$, but in the large- $\gamma$ limit the pressure is observed to localize in a box of length $O\left(\gamma^{-1}\right)$. In view of our normalization (2.6), this requires that the pressure for large $\gamma$ scales as $p \sim O\left(\gamma^{1 / 2}\right)$. This implies that the jump condition equations remain in balance if $\rho^{2} \sim \gamma^{-1 / 3}$, so that in the large- $\gamma$ limit we anticipate $\rho \gamma^{1 / 6}$ to be $O(1)$. More precisely, the above argument suggests that for large $\gamma$ the wave amplitude $(\rho)$ to wavenumber $(\alpha, \gamma)$ nonlinear eigenrelation takes on the functional form

$$
\rho \gamma^{1 / 6}=\left(\frac{\alpha}{\gamma}\right)^{5 / 6} S\left(\frac{\alpha}{\gamma}\right),
$$

where $(\alpha / \gamma)^{5 / 6}$ is the behaviour required for $(\alpha / \gamma) \rightarrow 0$ (cf. figure 5 of Hall \& Sherwin 2010) and function $S$ represents deviation from this scaling, further from the origin.

Our rescaled results for the data shown in figure 2 for $(\rho, \alpha)$ and $\gamma=5,6,7$ are replotted in figure 5 as functions of $\alpha / \gamma$ versus $\rho \gamma^{1 / 6}$. We observe that for these higher values of $\gamma$ there is a good collapse of the data. We have also explored even higher values of $\gamma$ where there is reasonable agreement with this collapse but we are unable to stabilize our hybrid algorithm for sufficiently high regularized Reynolds 


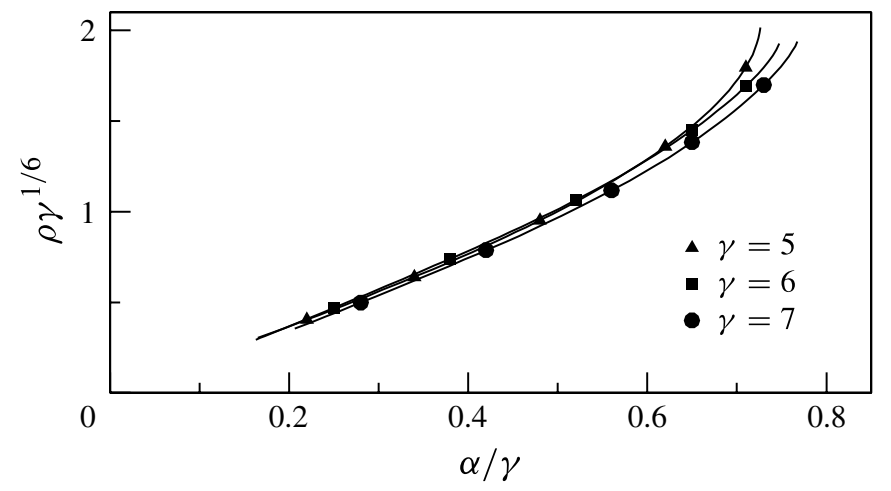

FIGURE 5. Collapse of the large- $\gamma$ nonlinear eigenrelation data from figure 2, in agreement with the relationship (4.2).

numbers to achieve the asymptotic solution implied by the inviscid wave equation. As discussed earlier, it is likely that, to extend our results to higher $\gamma$, an algorithm based on the smoother wave pressure is required in which it will be necessary to directly impose the stress jump conditions.

Now let us re-examine the consequences of these rescalings. If we make the above transformations and $\rho p \rightarrow \gamma^{-1 / 3} \rho p$, then the full interaction equations remain identical to (2.4), (2.7) and (2.8); the only difference is that, rather than imposing no slip at the rigid walls, the roll and streak must satisfy $(U, V, W) \rightarrow( \pm y, 0,0)$ as $y \rightarrow \pm \infty$. Our results then suggest the emergence of a new canonical class of lower branch equilibria for arbitrary shear flows. In terms of the original VWI equations, the rescaling in terms of $\gamma$ leads to exactly the same interaction equations. An examination of the form for the solution reveals that $d$, the height of the channel, now only appears in the form $d / \gamma$, i.e. a typical vortex wavelength. If we then define the Reynolds number based on a characteristic velocity near the centre, which becomes the speed of the plates multiplied by $d / \gamma$, and use $d / \gamma$ as a typical length scale, then $d$ is effectively scaled out of the problem. Rather than give the complete details for the rescaling of the Couette flow problem, it is more convenient to simply write down the canonical problem for quite arbitrary shear flows, as follows.

\subsection{Canonical vortex wave interactions in arbitrary shear flows}

Suppose that we have a shear flow $\boldsymbol{U}^{*}=\left(U^{*}\left(y^{*}\right), 0,0\right)$, where ${ }^{*}$ denotes a dimensional quantity, and that we are interested in VWI with dimensional spanwise wavelength $\lambda^{*}=2 \pi / \gamma^{*}$. Without loss of generality we seek a VWI solution near $y^{*}=0$ and define a Reynolds number by

$$
R=\partial_{y^{*}}\left(U_{0}^{*}\right) \lambda^{* 2} / \nu,
$$

i.e. we use $\partial_{y^{*}}\left(U_{0}^{*}\right) \lambda^{*}=\left.\partial_{y^{*}}\left(U^{*}\right)\right|_{y^{*}=0} \lambda^{*}$ as a typical flow speed. We then make the Navier-Stokes equations dimensionless using this typical flow speed and $\lambda^{*}$ as a typical length in (2.1). Equations (2.2) apply once again and we are led to the streak-roll equations (2.3), (2.4) and the wave equation (2.5). As discussed in $\S 4.1$, the main difference is that now in (2.3) the condition on $U$ must be applied at $y= \pm \infty$ where the wave must also vanish. Moreover, the streak-roll-wave is now periodic in the spanwise direction with wavelength $2 \pi$. We conclude that the VWI described in Hall \& Sherwin (2010) applies to arbitrary one-dimensional shear flows and describes states localized about some level. 


\section{Lower branch equilibria in Couette flow}

The fact that the streak-roll-wave is now confined to a layer small compared to the length scale over which the original flow changes has further important consequences. For example, if we next consider a more general initial shear flow $\boldsymbol{U}^{*}=\left(U^{*}\left(y^{*}, z^{*}\right), 0,0\right)$, then for a given real wave speed $c^{*}$ we can look for a VWI structure localized in a thin layer around the position where $U^{*}=c^{*}$. VWI states with wavenumber slowly varying along the critical layer can be constructed locally from the canonical state discussed above. If the critical layer is spatially closed, then periodicity will fix discrete nonlinear eigenvalues. Such a structure could be used to describe large-azimuthal-wavenumber VWI states for Hagen-Poiseuille flow, although this possibility is not investigated further here.

Now consider the energy density function of a wave travelling with the local speed of the flow at position $y^{*}$ for this localized problem. The energy per unit mass per unit wavenumber of the wave

$$
E \sim \lambda^{*} \boldsymbol{u}^{\prime} \cdot \boldsymbol{u}^{\prime} /(4 \pi)
$$

where $\lambda^{*}$ is a characteristic wavelength in the $x-z$ plane, inversely proportional to wavenumber. The viscous dissipation

$$
\Phi \sim \nu U_{y^{*}}^{2} / \lambda^{* 2},
$$

where $U_{y^{*}}=\partial_{y^{*}} U\left(y^{*}\right) \lambda^{*}$ is a typical flow speed and $v$ is kinematic viscosity. Adopting $\tilde{R}=U_{y^{*}} \lambda^{*} / v$ as the Reynolds number, we know that, away from the critical layer, the wave is of magnitude $U_{y^{*}} \tilde{R}^{-7 / 6}$, so that

$$
E \sim U_{y^{*}}^{2} \tilde{R}^{-7 / 3} \lambda^{*}=\left(U_{y^{*}}^{2} v^{2 / 3} v^{5 / 3} \lambda^{*}\right) /\left(U_{y^{*}}^{7 / 3} \lambda^{* 7 / 3}\right) .
$$

Substituting $v^{2 / 3}=\lambda^{* 4 / 3} \Phi^{2 / 3} / U_{y^{*}}^{4 / 3}$ above, we obtain

$$
E \sim \tilde{R}^{-5 / 3} \lambda^{* 5 / 3} \Phi^{2 / 3}
$$

This is the Kolmogorov 5/3 rule with the important caveat that the result is multiplied by a dimensionless quantity involving the wavelength. It is interesting to note the appearance of the Kolmogorov cascade scale, though the full significance of this is yet to be determined.

\section{Conclusion}

Building on evidence obtained from lower branch equilibrium VWI structures in plane Couette flow and using scaling arguments, we have two new outcomes for large-wavenumber and large-Reynolds-number applications of VWI theory: first, that it leads to self-similar equilibrium structures relevant to arbitrary shear flows, far from any bounding wall; and second, that the relationship between wave energy and dissipation rate of these structures reproduces a scaling similar to Kolmogorov's $-5 / 3$ law for turbulent flows (Kolmogorov 1941). Kolmogorov derived his result based on dimensional arguments concerning energy transfer at high wavenumbers, whereas our analysis examines a specific physical mechanism in a critical layer. Though we have considered a single wave, the theory can be extended over a sum of waves, with each wave driving shear stress discontinuities across its associated critical layer. In that case the $E \sim \lambda^{* 5 / 3}$ scaling describes the energy density necessary to maintain the local streak flow supporting the wave. As such, the vertical structure of the streak adjusts to make all the waves neutral, so different waves communicate through their local effect 


\section{H. M. Blackburn, P. Hall and S. J. Sherwin}

on the streak. This suggests that a relationship may exist between the $-5 / 3$ law and the critical layer structure of waves predicted by VWI theory.

\section{Acknowledgements}

H.M.B. and S.J.S. would like to acknowledge financial support, respectively, from Australian Research Council grant DP1094851 and EPSRC grant EP/H050507/1. P.H. and S.J.S. would also like to acknowledge support under EPSRC grant EP/I037946/1.

\section{Supplementary movies}

Supplementary movies are available at http://dx.doi.org/10.1017/jfm.2013.254.

\section{References}

Faisst, H. \& Eckhardt, B. 2003 Traveling waves in pipe flow. Phys. Rev. Lett. 91 (22), 224502.

Gibson, J. F., Halcrow, J. \& Cvitanovic, P. 2009 Equilibrium and travelling-wave solutions of plane Couette flow. J. Fluid Mech. 638, 243-266.

Hall, P. \& Horseman, N. J. 1991 The linear inviscid secondary instability of longitudinal vortex structures in boundary layers. J. Fluid Mech. 232, 357-375.

HALl, P. \& SHERWIN, S. J. 2010 Streamwise vortices in shear flows: harbingers of transition and the skeleton of coherent structures. J. Fluid Mech. 661, 178-205.

Hall, P. \& SMith, F. T. 1988 The nonlinear interaction of Tollmein-Schlichting waves and Taylor-Görtler vortices in curved channel flows. Proc. R. Soc. Lond. A 417, 255-282.

HALL, P. \& SMITH, F. T. 1991 On strongly nonlinear vortex/wave interactions in boundary-layer transition. J. Fluid Mech. 227, 641-666.

Hamilton, J. M., Kim, J. \& WalefFe, F. 1995 Regeneration mechanisms of near-wall turbulence structures. J. Fluid Mech. 287, 317-348.

Kolmogorov, A. N. 1941 Local structure of turbulence in incompessible viscous fluid at very large Reynolds numbers. Dokl. Akad. Nauk SSSR 30, 301-305.

NAGATA, M. 1990 Three-dimensional finite-amplitude solutions in plane Couette flow: bifurcation from infinity. J. Fluid Mech. 217, 519-527.

SCHNEIDER, T. M. \& ECKHARDT, B 2009 Edge states intermediate between laminar and turbulent states in pipe flow. Phil. Trans. R. Soc. Lond. A 367, 577-587.

Waleffe, F. 1997 On a self-sustaining process in shear flows. Phys. Fluids 9, 883-890.

WALEFFe, F. 2001 Exact coherent structures in channel flow. J. Fluid Mech. 435, 93-102.

WANG, J., GiBSON, J. F. \& WALEFFE, F. 2007 Lower branch coherent states in shear flows: transition and control. Phys. Rev. Lett. 98 (20), 204501.

Wedin, H. \& KeRswell, R. R. 2004 Exact coherent structures in pipe flow: travelling wave solutions. J. Fluid Mech. 508, 333-371. 